\title{
Knowledge about Colorectal Cancer in Northern Iran: a Population-Based Telephone Survey
}

\author{
Alireza Mansour-Ghanaei ${ }^{1 *}$, Farahnaz Joukar ${ }^{2 *}$, Fariborz Mansour-Ghanaei ${ }^{2 *}$, \\ Javid Rasoulian², Mohammad Reza Naghipour ${ }^{2}$, Arezoo Fani², Ehsan \\ Kazemnejad ${ }^{2}$
}

\begin{abstract}
Background: Colorectal cancer (CRC) is one of the most common malignancies of the gastrointestinal tract. The aim of this study was to assess the general knowledge of CRC in individuals living in Rasht, Iran, using a population-based cross-sectional telephone survey. Materials and Methods: A total of 1557 participants between 18 and 80 years of age were interviewed using random sampling from the telephone directory. Knowledge of risk factors, symptoms, diagnosis, and prevention of CRC was assessed using a validated questionnaire. Results: The mean knowledge level of the 1,557 respondents (average age $46 \mathrm{y}$ ) was $13.5 \pm 4.29$ (maximum possible score $=26)$, and $46.4 \%(722 / 1,557)$ of the subjects achieved grades lower than the mean score. The mean scores for knowledge of symptoms and risk factors were $3.97 \pm 1.83$ (range: $0-7$ ) and $5.17 \pm 1.65$ (range: $0-9$ ), respectively. Older age, higher education, and employment were significantly associated with better scores for recognition of risk factors and warning symptoms. The majority of subjects correctly identified weight loss $(70.2 \% ; 1,093 / 1,557)$ and rectal bleeding $(63.3 \%$; 986/1,557) as symptoms of CRC, and that smoking $(85.9 \% ; 1,337 / 1,557)$ and a lowfiber diet $(73.4 \% ; 1,143 / 1,557)$ were risk factors. Approximately half of the subjects noted increasing age, genetic background and fried food as other risk factors. A considerable number $(54.8 \% ; 853 / 1,557)$ identified colonoscopy as a screening method for detecting $\mathrm{CRC}$ in asymptomatic patients. However, a third of the subjects in the target group for screening ( $\geq 50 \mathrm{y}$ ) were not interested in undergoing screening, primarily due to a lack of symptoms. Conclusions: Our results suggest that the knowledge of CRC is poor among the public, and therefore greater attempts should be made to increase awareness. Public education emphasizing the risk factors and symptoms of CRC, as well as the importance of regular screening regardless of the presence of symptoms, may help to reduce CRC morbidity and mortality.
\end{abstract}

Keywords: Colorectal carcinoma - knowledge - population-based planning - Iran

Asian Pac J Cancer Prev, 16 (17), 7831-7836

\section{Introduction}

Cancer is among the most dangerous diseases worldwide, with an increasing number of cases and deaths (Kozlowska et al., 2011). Colorectal cancer (CRC) accounts for an estimated 1.2 million new cancer cases worldwide and over 630,000 cancer deaths $(\sim 8 \%)$ per year (Kamangar et al., 2006; Society, 2007), becoming the third most common cause of cancer-related death (Pourhoseingholi and Zali, 2012; Ng and Wong, 2013). The incidence of CRC has increased during the last 25 years (Azadeh et al., 2008), The most common cancer among Iranian women was breast cancer and among Iranian men was stomach cancer besides skin cancer. Other most common cancers were colorectal, stomach and esophagus in females. Bladder, prostate, colorectal and esophagus were common cancers among males (Mousavi et al., 2009). There is also a high frequency of a positive family history of CRC in Iranian patients, thus the incidence of this disease is likely to increase dramatically in the near future (Kolahdoozan et al., 2010).

Tumor stage at the time of presentation is the key prognostic factor for CRC survival, and thus, early identification is crucial (Syahnaz Mohd Hashim, 2011). However, delayed diagnosis of CRC is common, despite considerable advancements in diagnostic methods. Therefore, facilitating access to CRC screening is an important key to reducing the burden of CRC (Pourhoseingholi, 2012). Three screening modalities are frequently used, including fecal occult blood tests, flexible sigmoidoscopy and total colonoscopy (Sung, 2007; Brock et al., 2012; Joukar et al., 2012). CRC screening strategies are typically based on annual or biennial fecal occult blood tests, or endoscopic procedures performed once every five (sigmoidoscopy) or ten (colonoscopy) years (Gimeno Garcia, 2012).

${ }^{1}$ Medical Student at Shahid Beheshti University of Medical Sciences (SBMU), Tehran, ${ }^{2}$ Department of Gastroenterology, Gastrointestinal and Liver Diseases Research Center (GLDRC), Guilan University of Medical Sciences, Rasht, Iran *For correspondence:farajov@gmail.com,f_jokar@gums.ac.ir,ghanaie@yahoo.com 
Studies have shown that education level, annual income, inconvenient and embarrassing nature of the test, lack of physician recommendation, and knowledge of CRC are obstacles for implementing CRC screening programs (Wong et al., 2013; Gimeno Garcia et al., 2014). Basic public knowledge, including awareness of methods for prevention, early diagnosis, screening, and treatment, is important for controlling any kind of cancer. However, there is little information regarding the knowledge of CRC screening in Iran. The aim of the present study was to assess the knowledge of CRC among individuals living in Rasht, in northern Iran.

\section{Materials and Methods}

\section{Study design and participant selection}

A cross-sectional population-based telephone survey of residents of Rasht (the capital of Guilan province in northern Iran) was conducted between September 2011 and September 2012 by the Gastrointestinal and Liver Diseases Research Center of Guilan University of Medical Sciences (GLDRC). A sample size of 1557 people was calculated based on the results of a pilot group $(\mathrm{P}=10 \%)$ and considering the precision of 0.01 and the type I error of 0.05 . The first telephone number from the Rasht telephone directory was selected randomly, and every 80th number was selected to obtain a total of 1557 numbers. A total of 2480 individuals were contacted by telephone to assess eligibility and interest in the study. Of these, 923 $(37.2 \%)$ were not interested ; which included $607 \mathrm{men}$ and 316 women. All of the interviews were made by two, trained general practitioners. The objective of the survey was explained to the responders and the participants were assured of the confidentiality of the survey. Only participants $\geq 18$ years of age were included, and those who did not provide consent or failed to complete the interview were excluded from the study.

\section{Questionnaire}

A pilot survey was first conducted with approximately twenty randomly selected telephone numbers to assess the ease of understanding and reliability of the language of the questionnaire. Minor modifications to the wording and format were made based on the pilot. The questionnaire's reliability was also confirmed by Cronbach's alpha coefficient (alpha $=0.7$ ). Content validity was examined and the questionnaire was revised based on the scores provided by seven experts. The survey was comprised of a simple, easy to understand, three-part questionnaire designed to determine the respondents' biographic data and knowledge of CRC.

The first part of the questionnaire involved sociodemographic information, including the age, sex, marital status, education, occupational status and information about patient with Gastrointestinal (GI) cancer and nonGI cancers of the participants. Previous medical history , family history of GI diseases and history of screening tests such as Pap smear, mammography and colonoscopy were also recorded. In addition, the participants were asked to name of the three top causes for cancer mortality in Iran which are Gastrointestinal, Lung and breast cancers
(Abazari et al., 2014).

The second part of the questionnaire assessed the participants' knowledge of CRC symptoms (seven items: rectal bleeding, abdominal pain, decreased appetite, change in bowel habit, weight loss, fever, and palpable mass in the abdomen), risk factors (nine items: hemorrhoid, fried food, genetic, low-fiber diet, stress, age, smoking, in men more than women, and not age-related), diagnosis (seven items: fecal occult blood test, blood test, colonoscopy, chest X-ray, genetic tests, colonoscopy before symptoms, and fecal occult blood test before symptoms), and prevention (three items: regular exercise, high-fiber diet and undergo colonoscopy screening). Suggested correct answers are determined at Table 2. Knowledge items were given a value of 1 if a respondent correctly answered "true" or "false," and 0 if the respondent answered incorrectly or was "unsure" (maximum score $=26$ ), and the mean knowledge score was determined for each demographic group.

The third part of the questionnaire assessed if participants had previously undergone screening for any type of cancer. A standardized, lay description of each test was presented to ensure adequate comprehension and correct representation. In addition, respondents who were $>50 \mathrm{y}$ of age were asked about the most important factor preventing them from undergoing colonoscopic screening. Also, those aware of the CRC and screening tests were asked about the method of notification.

\section{Statistical analysis}

Collected data were entered and analyzed using the Statistical Package for the Social Sciences (SPSS) for Windows version 19 (SPSS Inc., Chicago, IL, USA). Preliminary descriptive and bivariate analyses were performed, and knowledge scores of symptoms, risk factors, diagnosis and prevention, as well as their associations with independent variables were evaluated using analyses of variance. A $\mathrm{P}<0.05$ was considered significant.

\section{Results}

A total of 1557 participants were interviewed, of which $65.1 \%(1013 / 1557)$ were female (Table 1$)$. The vast majority of participants were married $(80.2 \%$; 1249/1557), had completed at least a high school education $(96.7 \%$; $1505 / 1557)$, and were not currently employed (77.3\%; 1204/1557).

The knowledge of cancer was assessed by asking the participants to name the three top causes for cancer mortality in Iran. A large percentage $(27.9 \%$; 434/1557) of the respondents accurately identified at least one, however, only $1.0 \%(16 / 1557)$ were able to correctly name all three. Overall, the mean knowledge of CRC was $13.50 \pm 4.29$, with $46.4 \%(722 / 1557)$ achieving a score lower, and $53.6 \%(835 / 1557)$ higher than the mean score. Participants most frequently attributed their knowledge of cancer to radio and TV $(48.0 \%$; 748/1557). Participants less commonly attributed knowledge to magazine articles and only rarely reported their physicians as their source of information. 
Participants $>40 \mathrm{y}$ of age had significantly higher knowledge scores for risk factors and signs and symptoms (Ps < 0.01) (Table 1), and compared to those $<40 \mathrm{y}$, more often identified weight loss as a risk factor (73.0 vs.69.0\%). The risk factor for CRC that was least frequently identified was hemorrhoids, with only $23 \%$ $(368 / 1557)$ of respondents recognizing this as a risk, whereas $85.9 \%(1337 / 1557)$ were aware that smoking is a
DOI:http://dx.doi.org/10.7314/APJCP.2015.16.17.7831 Knowledge about Colorectal Carcinoma in Rasht, Northern Iran risk factor (Table 2). Women were equally knowledgeable of signs and symptoms, but significantly more likely than men to recognize weight loss $(72.1$ vs $66.7 \% ; \mathrm{P}=0.02)$ and decreased appetite (63.6 vs 54.0\%; $\mathrm{P}<0.01)$.

Of the 1557 participants, $10 \%$ (156) had previously participated screening for any type of cancer, though < $1 \%(16 / 156)$ had undergone a colonoscopy. Three hundred eighty-two respondents (226 female, 156 male) were $\geq 50$

Table 1. Knowledge Scores According to Demographic Characteristics

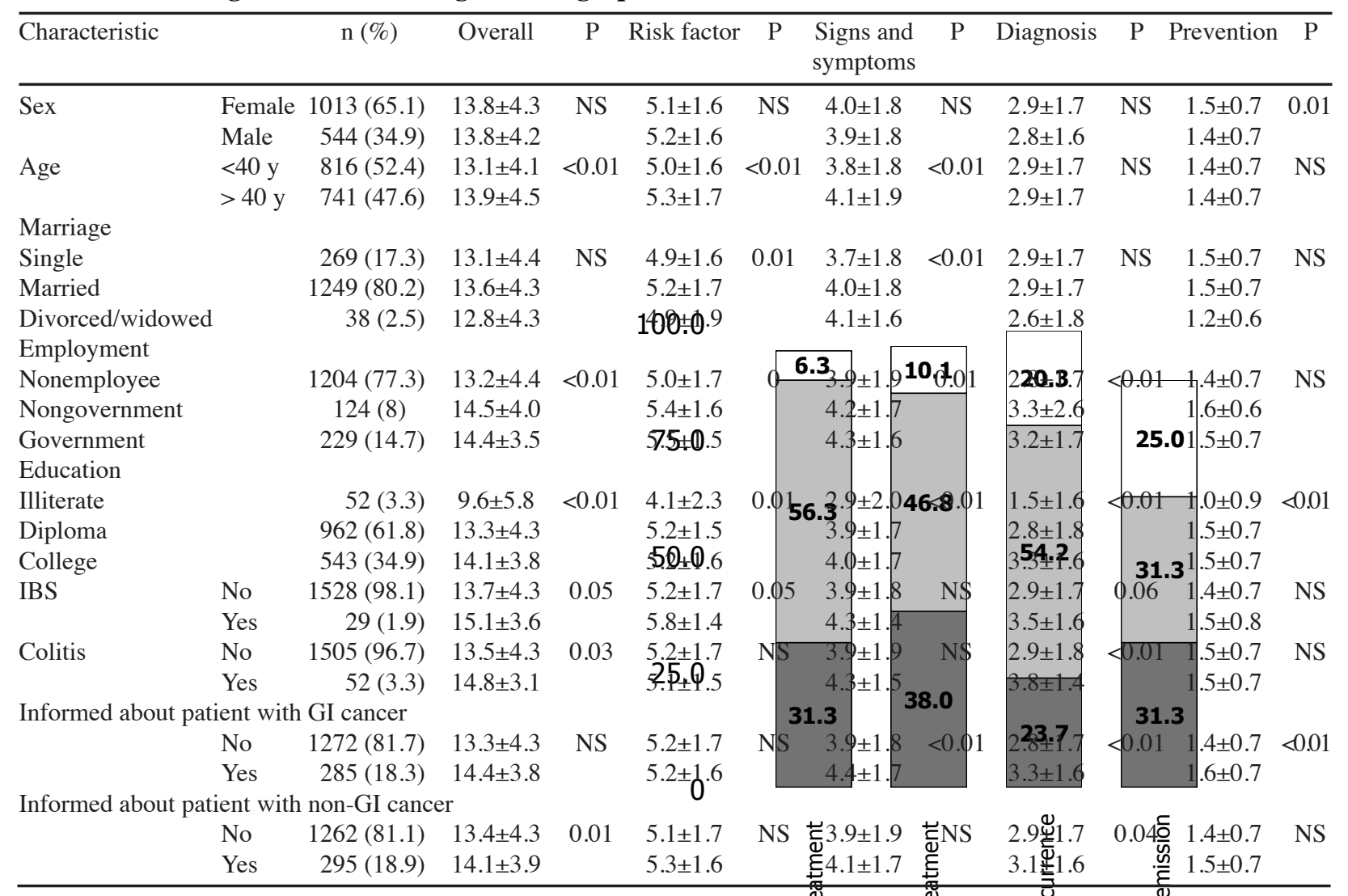

Table 2. Correct Responses According to Gender

\begin{tabular}{|c|c|c|c|c|}
\hline \multirow{2}{*}{$\begin{array}{l}\text { Statement about } \\
\text { colorectal carcinoma }\end{array}$} & & \multicolumn{2}{|c|}{ Correct response, n (\%) } & \multirow[t]{2}{*}{$\mathrm{P}$ value } \\
\hline & & Female & Male & \\
\hline \multirow[t]{9}{*}{ Risk factor } & Hemorrhoid** & $197(19.4)$ & $171(31.4)$ & $<0.01$ \\
\hline & Fried Food* & $709(70.0)$ & $354(65.1)$ & 0.04 \\
\hline & Genetic* & $665(65.6)$ & $333(61.2)$ & NS \\
\hline & Low-fiber diet* & $763(75.3)$ & $380(69.9)$ & 0.02 \\
\hline & Stress $* *$ & $747(73.7)$ & $386(71.0)$ & NS \\
\hline & Age* & $595(58.7)$ & $358(65.8)$ & 0.01 \\
\hline & Smoking* & $881(87.0)$ & $456(83.8)$ & NS \\
\hline & In men more than women* & $457(45.1)$ & $328(60.3)$ & $<0.01$ \\
\hline & History of GI disorder & $178(17.6)$ & $98(18.0)$ & NS \\
\hline \multirow[t]{7}{*}{ Signs and symptoms } & Rectal bleeding* & $640(63.2)$ & $346(63.6)$ & NS \\
\hline & Abdominal pain* & $639(63.1)$ & $341(62.7)$ & NS \\
\hline & Decreased appetite* & $644(63.6)$ & $294(54.0)$ & $<0.01$ \\
\hline & Change in bowel habit* & $586(57.8)$ & $283(52.0)$ & NS \\
\hline & Weight loss $*$ & $730(72.1)$ & $363(66.7)$ & 0.02 \\
\hline & Fever** & $198(19.5)$ & $153(28.1)$ & $<0.01$ \\
\hline & Palpable mass in the abdomen* & $616(60.8)$ & $351(64.5)$ & NS \\
\hline \multirow[t]{5}{*}{ Diagnosis } & Fecal occult blood test* & $593(58.5)$ & $341(62.7)$ & NS \\
\hline & Blood test $* *$ & $132(13.0)$ & $78(14.3)$ & NS \\
\hline & Colonoscopy* & $676(66.7)$ & $315(57.9)$ & $<0.01$ \\
\hline & Chest X-ray** & $441(43.5)$ & $261(48.0)$ & NS \\
\hline & Genetic tests* & $160(15.8)$ & $104(19.1)$ & NS \\
\hline
\end{tabular}

Items contain (*) are suggested as correct (Yes) answers and (**) are suggested as incorrect answers 
$\mathrm{y}$ of age, and were therefore considered a target group for screening. When asked if they would undergo screening as an asymptomatic patient in order to detect early cancer, $68.1 \%$ (260/382) reported that they would comply with the screening test. Participants were then asked to select which factors would negatively impact their compliance with colonoscopic investigations. The most common $(37.1 \% ; 142 / 382)$ response was that the respondents believed that screening was unnecessary because they were asymptomatic. Other reported factors included the cost of the screening method $(32.9 \% ; 126 / 382)$, and pain $(15.2 \% ; 58 / 382)$ and embarrassment $(3.9 \% ; 15 / 382)$ involved, while others $(25.9 \%$; 99/382) claimed they had no reason for not undergoing screening.

\section{Discussion}

Understanding and recognizing public awareness for CRC may provide valuable information for policy decisions for prevention, early diagnosis and improvement of survival. Worldwide, a substantial body of evidence indicates that there is a minimal knowledge of CRC in many countries. The Asia Pacific Working Group in Colorectal Cancer conducted a multinational survey in various Asia Pacific regions and found that the median symptom and risk factor knowledge scores ranged from 0-4 out of 9, with a number of regions scoring 0 (Koo et al., 2012). Another survey among moderate-risk patients in West Malaysia reported that only $4.1 \%$ had adequate knowledge of CRC and its screening (Harmy et al., 2011). Other studies on CRC knowledge among indigenous Western Australians (Christou and Thompson, 2012), Iranians (Salimzadeh et al., 2012), American Indians (Sanderson et al., 2011), and ethnically diverse populations in Australia (Javanparast et al., 2012) and Hong Kong (Tam et al., 2011), also found minimal knowledge of CRC, including awareness of its symptoms and risk factors.

Awareness of risk factors for CRC in the Iranian population in this study was adequate when participants were asked to recognize risk factors, such as family history and a diet low in fiber and vegetables. In a study by Su et al (2013), only $11.6 \%$ correctly identified diet as a factor, whereas $77 \%$ percent of respondents could not recall any risk factors without aid. In addition, the majority of participants in the present study correctly reported that adults over 50 years of age were at greater risk, and those in the older age group were significantly more knowledgeable about CRC. Furthermore, education and a history of irritable bowel syndrome were associated with higher knowledge scores, consistent with previous works (Yardley et al.,2000; Robb et al., 2009; Bidouei et al., 2014). Associations between knowledge and socioeconomic variation were also identified, corresponding with the socioeconomic gradient in bowel cancer screening that has been consistently reported (McCaffery et al., 2002). As mentioned in a prior study in Iran, positive family history of CRC did not increase the knowledge of our population (Bidouei et al., 2014), we could not assess this association due to the small number of participants with a positive family history.
The results of this study indicate there is much room for improvement concerning the awareness of CRC risk factors and symptoms. Ignorance, embarrassment, and fear may all contribute to patient delay in seeking advice about CRC symptoms (Yardley et al., 2000). In the study by Ray et al. (2004), 44\% of the respondents knew the major risk factors of cancer, and only $35 \%$ knew the primary symptoms. In a study by Puri et al. (2010), 78.6\% of Indian respondents knew that cancer can be symptomatic, and most commonly identified unusual swelling and the presence of blood in vomit as symptoms. Cachexic features were known to less than half of respondents, whereas weight loss was the most common symptom identified by our respondents. This is in contrast to results from a study by Yardley et al. (2000) that reported only $31 \%$ of respondents in the UK were able to state a symptom of CRC. More than $90 \%$ of respondents in a study by McCaffery et al. (2003) stated that they would visit their general practitioner if they noticed bleeding, and $77 \%$ would if they noticed a persistent change in bowel habits. In contrast, only $63.3 \%$ of participants in our study recognized bowel bleeding as a symptom of CRC and $55.8 \%$ associated change of bowel habit with CRC. Although there is evidence that bleeding may be the better predictor of bowel cancer, the findings suggest that more education regarding changes in bowel habits as an early symptom may be needed (Starmans et al., 1994; Muris et al., 1995).

Smoking was correctly identified as a risk factor by a vast majority of our participants, consistent with findings reported by Puri et al. (2010) and Bhurgri et al. (2008). Undoubtedly, the burden due to smoking-related cancers is increasing throughout the world and tobacco control would be a cost-effective method for cancer prevention. Physical inactivity has also been associated with CRC, as it increases bowel transit time, and thus, the duration of contact between fecal carcinogens and colon mucosa (Batty and Thune, 2000). Despite the well-published benefits, many individuals are not sufficiently engaged in physical activity, and the majority of participants in our study had no knowledge that exercise protects against the development of CRC.

In the present study, the majority of the respondents were aware of colonoscopy and fecal occult blood tests as CRC screening and diagnostic tests. These results are in contrast to studies by Wong et al. (2006) and Harmy et al. (2011), where the majority were unaware of these methods. However, the absence of clinical symptoms as the most frequently reported explanation for ignoring CRC screening tests is consistent with previous studies (Wolf et al., 2001; Hoffman et al., 2011; Salimzadeh et al., 2012). In addition, the lack of physician recommendations is a commonly reported barrier to obtaining CRC screening tests in Tehran (Salimzadeh et al., 2011; Salimzadeh et al., 2012) and Shiraz (Roozitalab et al., 2012). Other barriers, such as fear of the detection of cancer and cost of tests, have been reported less often in these studies (Rawl et al., 2005; Green et al., 2008; Omran and Ismail, 2010).

Similar to other studies (Salimzadeh et al., 2012; Bidouei et al., 2014), there was no significant difference between men and women in overall CRC knowledge 
and prior screening. The participants in our study mainly attributed their knowledge of cancer to radio and television, which is equally available to men and women in this area. Family physicians are one of the most important sources of health education for the general public who can provide information and techniques to help patients take control of their health and focus on preventing illness. The lack of a suitable family physician program in Guilan province, which has a relatively high prevalence of gastrointestinal cancer (Harmy et al., 2011), is likely one of the important causes for the minimal knowledge and interest for screening in this area.

Despite important information obtained from this study, a possible limitation is the fact that subjects were asked to recognize specific items concerning CRC from a written list. Although recognition scores tend to be higher than recall scores, it is hard to determine which is a better predictor of cancer knowledge and awareness. Recall is limited by memory and perseverance, and may underestimate awareness, whereas recognition encourages guessing and hence overestimates awareness.

The results of the current study suggest that community knowledge levels concerning some aspects of CRC are relatively low. In order to promote CRC screening, public education should be intensified to increase awareness of CRC, and the effectiveness of early screening for prevention and potential cures. General practitioners are an important source of knowledge and should encourage CRC screening. Future research should evaluate what educational interventions are most effective and feasible for people at higher risks for poor knowledge of CRC. In addition, similar surveys should be conducted in various population groups to compare findings.

\section{Acknowledgements}

We would like to thank all the Gastrointestinal \& Liver Diseases Research Center (GLDRC) personnel, especially Miss. Maryam Moradi, who assisted us in reporting this study.

\section{References}

Abazari M, Gholamnejad M, Roshanaei G, et al (2014). Estimation of survival rates in patients with lung cancer in west azerbaijan, the northwest of iran. Asian Pacific journal of cancer prevention: APJCP, 16, 3923-6.

Azadeh S, Moghimi-Dehkordi B, Fatem SR, et al (2008). Colorectal cancer in Iran: an epidemiological study. Asian Pac J Cancer Prev, 9, 123-6.

Batty D, Thune I (2000). Does physical activity prevent cancer?: Evidence suggests protection against colon cancer and probably breast cancer. BMJ: British Medical Journal, 321, 1424.

Bhurgri H, Gowani SA, Itrat A, et al (2008). Awareness of cancer risk factors among patients and attendants presenting to a tertiary care hospital in Karachi, Pakistan. Journal of the Pakistan Medical Association, 58, 584.

Bidouei F, Abdolhosseini S, Jafarzadeh N, et al (2014). Knowledge and perception toward colorectal cancer screening in east of Iran. International journal of health policy and management, $\mathbf{3}, 11$.

Brock R, Xiong B, Li L, et al (2012). A multiplex serum protein
DOI:http://dx.doi.org/10.7314/APJCP.2015.16.17.7831

Knowledge about Colorectal Carcinoma in Rasht, Northern Iran assay for determining the probability of colorectal cancer. Am J Cancer Res, 2, 598-605.

Christou A, Thompson SC (2012). Colorectal cancer screening knowledge, attitudes and behavioural intention among Indigenous Western Australians. BMC public health, 12, 528.

Gimeno Garcia AZ (2012). Factors influencing colorectal cancer screening participation. Gastroenterol Res Pract, 2012, 483417.

Gimeno Garcia AZ, Hernandez Alvarez Buylla N, NicolasPerez D, et al (2014). Public awareness of colorectal cancer screening: knowledge, attitudes, and interventions for increasing screening uptake. ISRN Oncol, 2014, 425787.

Green AR, Peters-Lewis A, Percac-Lima S, et al (2008). Barriers to screening colonoscopy for low-income Latino and white patients in an urban community health center. J general internal medicine, $\mathbf{2 3}, 834-40$.

Harmy M, Norwati D, Noor NM, et al (2011). Knowledge and attitude of colorectal cancer screening among moderate risk patients in West Malaysia. Asian Pac J Cancer Prev, 12, 1957-60.

Hoffman RM, Rhyne RL, Helitzer DL, et al (2011). Barriers to colorectal cancer screening: physician and general population perspectives, New Mexico, 2006. Preventing chronic disease, 8 , 1-11.

Javanparast S, Ward PR, Carter SM, et al (2012). Barriers to and facilitators of colorectal cancer screening in different population subgroups in Adelaide, South Australia. Med $J$ Aust, 196, 521-3.

Joukar F, Majd SK, Fani A, et al (2012). Colonoscopy outcome in North of Iran (Guilan): 2006-2009. Int J Clin Exp Med, 5,321-5.

Kamangar F, Dores GM,Anderson WF (2006). Patterns of cancer incidence, mortality, and prevalence across five continents: defining priorities to reduce cancer disparities in different geographic regions of the world. J Clin Oncol, 24, 2137-50.

Kolahdoozan S, Sadjadi A, Radmard AR, et al (2010). Five common cancers in Iran. Arch Iran Med, 13, 143-6.

Koo JH, Leong RW, Ching J, et al (2012). Knowledge of, attitudes toward, and barriers to participation of colorectal cancer screening tests in the Asia-Pacific region: a multicenter study. Gastrointestinal Endoscopy, 76, 126-35.

Kozlowska E, Szewczyk MT, Banaszkiewicz Z, et al (2011). Knowledge of symptoms and diagnostic possibilities of cancer diseases. Arch Med Sci, 7, 304-9.

McCaffery K, Wardle J, Nadel M, et al (2002). Socioeconomic variation in participation in colorectal cancer screening. $J$ Med Screen, 9, 104-8.

McCaffery K, Wardle J, Waller J (2003). Knowledge, attitudes, and behavioral intentions in relation to the early detection of colorectal cancer in the United Kingdom. Preventive Medicine, 36, 525-35.

Mousavi SM, Gouya MM, Ramazani R, et al (2009). Cancer incidence and mortality in Iran. Ann Oncol, 20, 556-63.

Muris J, Starmans R, Fijten GH, et al (1995). Non-acute abdominal complaints in general practice: diagnostic value of signs and symptoms. British J General Practice, 45, 313-6.

$\mathrm{Ng} \mathrm{SC}$, Wong SH (2013). Colorectal cancer screening in Asia. Br Med Bull, 105, 29-42.

Omran S, Ismail AA (2010). Knowledge and Beliefs of Jordanians Toward Colorectal Cancer Screening. Cancer nursing, 33, 141-8.

Pourhoseingholi MA (2012). Increased burden of colorectal cancer in Asia. World J Gastrointest Oncol, 4, 68-70.

Pourhoseingholi MA, Zali MR (2012). Colorectal cancer screening: Time for action in Iran. World J Gastrointest Oncol, 4, 82-3. 


\section{Javid Rasoulian et al}

Puri S, Mangat C, Bhatia V, et al (2010). Knowledge of cancer and its risk factors in Chandigarh, India. Int J Epidemiol, 8.

Rawl S, Menon U, Champion V, et al (2005). Do benefits and barriers differ by stage of adoption for colorectal cancer screening? Health Educ Res, 20, 137-48.

Ray K MS (2004). Knowledge about cancer in West Bengal- a pilot survey. Asian Pac J cancer Prev, 5, 205-12.

Robb K, Stubbings S, Ramirez A, et al (2009). Public awareness of cancer in Britain: a population-based survey of adults. British Journal of Cancer, 101, 18-23.

Roozitalab M, Moatari M, Gholamzadeh S, et al (2012). The effect of health belief on participation of the official administrative personnel in colorectal cancer screening programs in Shiraz University of Medical Sciences: 2004. Govaresh, 13, 19-24.

Salimzadeh H, Delavari A, Montazeri A, et al (2012). Knowledge and practice of iranians toward colorectal cancer, and barriers to screening. International J Preventive Medicine, 3, 29.

Salimzadeh H, Eftekhar H, Majdzadeh R, et al (2011). More than half of senior residents in Tehran have never heard about colorectal cancer screening. Asian Pac J Cancer Prev, 12, 2851-6.

Sanderson PR, Weinstein N, Teufel-Shone N, et al (2011). Peer reviewed: assessing colorectal cancer screening knowledge at tribal fairs. Preventing chronic disease, $\mathbf{8}$.

Society AC (2007). Cancer Facts and Figures 2007. Atlanta, GA: American Cancer Society.

Starmans R, Muris JW, Fijten GH, et al (1994). The diagnostic value of scoring models for organic and non-organic gastrointestinal disease, including the irritable-bowel syndrome. Medical Decision Making, 14, 208-14.

Su TT, Goh JY, Tan J, et al (2013). Level of colorectal cancer awareness: a cross sectional exploratory study among multiethnic rural population in Malaysia. BMC cancer, 13, 376.

Sung J (2007). Colorectal cancer screening: its time for action in Asia. Cancer Detect Prev, 31, 1-2.

Syahnaz Mohd Hashim TSF, Khairani Omar, Mohd Radzniwan Abdul Rashid, Shah SZ, Sagap I (2011). Knowledge of colorectal cancer among patients presenting with rectal bleeding and its association with delay in seeking medical advice. Asian Pac J Cancer Prev, 12, 2007-11.

Tam TK, Ng K, Lau C, et al (2011). Faecal occult blood screening: knowledge, attitudes, and practice in four Hong Kong primary care clinics. Hong Kong Med J, 17, 350-7.

Wolf RL, Zybert P, Brouse CH, et al (2001). Knowledge, beliefs, and barriers relevant to colorectal cancer screening in an urban population: a pilot study. Family \& community health, 24, 34-47.

Wong BC, Chan AO, Wong WM, et al (2006). Attitudes and knowledge of colorectal cancer and screening in Hong Kong: A population based study. Journal of gastroenterology and hepatology, 21, 41-6.

Wong MC, Hirai HW, Luk AK, et al (2013). The knowledge of colorectal cancer symptoms and risk factors among 10,078 screening participants: are high risk individuals more knowledgeable? PLoS One, 8, 60366.

Yardley C, Glover C, Allen-Mersh T (2000). Demographic factors associated with knowledge of colorectal cancer symptoms in a UK population-based survey. Annals of the Royal College of Surgeons of England, 82, 205. 